\title{
Linear Partial Differential Equations of First Order as Bi-Dimensional Inverse Moments Problem
}

\section{Maria Beatriz Pintarelli}

Facultad de Ingenieria, Universidad Nacional de La Plata, La Plata, Argentina

Email: mariabpintarelli@gmail.com

Received 29 March 2015; accepted 30 May 2015; published 2 June 2015

Copyright (C) 2015 by author and Scientific Research Publishing Inc.

This work is licensed under the Creative Commons Attribution International License (CC BY).

http://creativecommons.org/licenses/by/4.0/

(c) () Open Access

\section{Abstract}

We consider linear partial differential equations of first order $a(x, t) w_{x}(x, t)+b(x, t) w_{t}(x, t)=h(x, t) w(x, t)+r(x, t)$ on a region $E=\left(a_{1}, b_{1}\right) \times\left(a_{2}, b_{2}\right)$. We will see that we can write the equation in partial derivatives as an Fredholm integral equation of the first kind and will solve this latter with the techniques of inverse problem moments. We will find an approximated solution and bounds for the error of the estimated solution using the techniques on problem of moments.

\section{Keywords}

\section{Linear PDEs, Freholm Integral Equations, Generalized Moment Problem}

\section{Introduction}

We consider linear partial differential equation of first order of the general form:

$$
a(x, t) w_{x}(x, t)+b(x, t) w_{t}(x, t)=h(x, t) w(x, t)+r(x, t)
$$

where the unknown function $w(x, t)$ is defined in $E=\left(a_{1}, b_{1}\right) \times\left(a_{2}, b_{2}\right)$. We will consider Dirichlet conditions on the boundary $S=\partial E$ and $a(x, t), b(x, t), h(x, t)$ and $r(x, t)$ are known functions.

Equation (1) is a particular case of the quasi-linear equation

$$
a(x, t, w) w_{x}(x, t)+b(x, t, w) w_{t}(x, t)=h(x, t, w) \quad a, b, h \in C^{1}(E) .
$$

The conventional method to solve this equation is reduced to find all surfaces $z=w(x, t)$ that satisfy the 
above equation. This equation expresses that the tangent to a curve on the surface $z=w(x, t)$ is proportional to $(a, b, h)$. The solution of the quasi-linear equation can therefore be expressed by

$$
\frac{\mathrm{d} x}{\mathrm{~d} s}=a(x, t, w) \quad \frac{\mathrm{d} t}{\mathrm{~d} s}=b(x, t, w) \quad \frac{\mathrm{d} z}{\mathrm{~d} s}=h(x, t, w)
$$

where $r(s)=x(s) \hat{i}+t(s) \hat{j}+z(s) \hat{k}$ is a parametric curve belonging to the solution surface. Then we must solve a system of three simultaneous differential equations of the first order.

The general solution of this system of three equations consists of families of curves which are described by a system of three parametric equations with three arbitrary constants determined by initial conditions. This system is generally not linear and it is known that a system of non linear ordinary differential equations is difficult to solve explicitly. In general, geometrically in $R^{3}$, the curves are determined by at least two intersecting surfaces transversely. This can be accomplished, for example, eliminating the parameter $s$ and obtain

$$
w_{1}(x, t, w)=c_{1} \quad w_{2}(x, t, w)=c_{2}
$$

where $c_{1}$ and $c_{2}$ are arbitrary constants. The general solution will be

$$
w_{2}=\varphi\left(w_{1}\right)
$$

where $\varphi$ is an arbitrary function of $w_{1}$. For a particular solution you can find the function $\varphi$ de modo que $w_{2}=\varphi\left(w_{1}\right)$ so to satisfy $f_{1}(x, t, z)=0$ y $f_{2}(x, t, z)=0$.

We will show that, the partial differential Equation (1) can be transformed into a integral equation and that this one can be numerically solved using techniques normally employed with generalized moment problems [1]-[3]. This approach was already suggested by Ang [4] in relation with the heat conduction equation and we have applied to the non linear Klein-Gordon equation [5].

Next section is devoted to show how the differential Equation (1) is transformed into integral equation of first kind that can be seen as generalized moments problem as is shown in Section 3. There we also proof a theorem that guarantees under certain conditions the stability and convergence of the finite generalized moment problem. In Section 4, we exemplify the general method by applying it to some linear PDEs which are particular cases of Equation (1). Finally in Section 5, the method is applied to solve an equation of Klein Gordon with boundary conditions in a rectangular region.

The d-dimensional generalized moment problem [1] [2] can be posed as follows: find a function $u$ on a domain $\Omega \subset \mathbf{R}^{d}$ satisfying the sequence of equations

$$
\int_{\Omega} u(x) g_{n}(x) \mathrm{d} x=\mu_{n} \quad n \in \mathbf{N}
$$

where $\left(g_{n}\right)$ is a given sequence of functions lying in $\mathbf{L}^{2}(\Omega)$ linearly independent.

Many inverse problems can be formulated as an integral equation of the first kind, namely,

$$
\int_{a}^{b} K(x, y) u(y) \mathrm{d} y=f(x) \quad x \in(a, b)
$$

$K(x, y)$ and $f(x)$ are given functions and $u(y)$ is a solution to be determined, $f(x)$ is a result of experimental measurements and hence is given only at finite set of points. It follows that the above integral equation is equivalent to the following moment problem

$$
\int_{a}^{b} K\left(x_{n}, y\right) u(y) \mathrm{d} y=f\left(x_{n}\right) \quad n=1,2, \cdots
$$

Also we consider the multidimensional moment problems

$$
\int_{\Omega} K\left(x_{n}, y\right) u(y) \mathrm{d} y=f\left(x_{n}\right) \quad n=1,2, \cdots, \Omega \subset \mathbf{R}^{d} .
$$

Moment problem are usually ill-posed [6] [7]. There are various methods of constructing regularized solutions, that is, stable appoximate solutions with respect to the given data $\mu_{n}$. One of them is the method of truncated expansion [4].

The method of truncated expansion consists in approximating (5) by finite moment problems

$$
\int_{\Omega} u(x) g_{i}(x) \mathrm{d} x=\mu_{i} \quad i=1,2, \cdots, n .
$$


Solved in the subspace $\left\langle g_{1}, g_{2}, \cdots, g_{n}\right\rangle$ generated by $g_{1}, g_{2}, \cdots, g_{n}$ (6) is stable. Considering the case where the data $\mu=\left(\mu_{1}, \mu_{2}, \cdots, \mu_{n}\right)$ are inexact, we apply some convergence theorems and error estimates for the regularized solutions.

\section{Linear Partial Differential Equations of First Order as Integral Equations of First Kind}

Let $F(w(x, t))=0$ be a partial differential equations such as (1). The solution $w(x, t)$ is defined on the region $E=\left(a_{1}, b_{1}\right) \times\left(a_{2}, b_{2}\right)$ and verifies Dirichlet conditiones on the boundary $C=\partial E$ :

$$
\begin{gathered}
w\left(a_{1}, t\right)=s_{1}(t) \quad w\left(b_{1}, t\right)=s_{2}(t) \\
w\left(x, a_{2}\right)=s_{3}(x) \quad w\left(x, b_{2}\right)=s_{4}(x)
\end{gathered}
$$

Let $F^{*}=\left(F_{1}(w), F_{2}(w)\right)$ be a vectorial field such that $w$ verifies $\operatorname{div}\left(F^{*}\right)=h^{*}(w)$ with $h^{*}$ a known function and, reciprocally, if $w$ verifies $\operatorname{div}\left(F^{*}\right)=h^{*}(w)$ then $F(w(x, t))=0$.

Let $u(x, t, \tau, \xi)$ be the auxiliary function such that

$$
\nabla u=\left(u k_{1}(x, t, \tau, \xi), u k_{2}(x, t, \tau, \xi)\right) .
$$

Since

$$
\operatorname{udiv}\left(F^{*}\right)=u h^{*}(w)
$$

we have

$$
\iint_{E} u \operatorname{div}\left(F^{*}\right) \mathrm{d} A=\iint_{E} u h^{*}(w) \mathrm{d} A
$$

Moreover, as

$$
\operatorname{udiv}\left(F^{*}\right)=\operatorname{div}\left(u F^{*}\right)-F^{*} \cdot \nabla u
$$

and

$$
\iint_{E} u \operatorname{div}\left(F^{*}\right) \mathrm{d} A=\underbrace{\iint_{E} \operatorname{div}\left(u F^{*}\right) \mathrm{d} A}_{=\int_{C}\left(u F^{*}\right) \cdot n d s}-\iint_{E} F^{*} \cdot \nabla u \mathrm{~d} A
$$

we obtain

where $\nabla u=\left(u_{\tau}, u_{\xi}\right)$.

$$
\iint_{E} u h^{*}(w) \mathrm{d} A=\int_{C}\left(u F^{*}\right) \cdot n \mathrm{~d} s-\iint_{E} F^{*} \cdot \nabla u \mathrm{~d} A
$$

Then (7) gives:

$$
\iint_{E} u h^{*}(w) \mathrm{d} A+\iint_{E} F^{*} \cdot \nabla u \mathrm{~d} A=\int_{C}\left(u F^{*}\right) \cdot n \mathrm{~d} s
$$

and

$$
\iint_{E} u h^{*}(w) \mathrm{d} A+\iint_{E} F^{*} \cdot \nabla u \mathrm{~d} A=\iint_{E}\left(u h^{*}(w)+F^{*} \cdot \nabla u\right) \mathrm{d} A=\iint_{E}\left(u h^{*}(w)+F_{1} u_{\tau}+F_{2} u_{\xi}\right) \mathrm{d} A .
$$

Then

$$
\int_{a_{1}}^{b_{1}} \int_{a_{2}}^{b_{2}} u\left(h^{*}(w)+\sum_{i=1}^{2} F_{i}(w(\tau, \xi)) k_{i}(x, t, \tau, \xi)\right) \mathrm{d} \xi \mathrm{d} \tau=G(x, t)
$$

where

$$
\begin{aligned}
G(x, t)= & \int_{a_{2}}^{b_{2}}\left(u\left(x, t, b_{1}, \xi\right) F_{1}\left(w\left(b_{1}, \xi\right)\right)-u\left(x, t, a_{1}, \xi\right) F_{1}\left(w\left(a_{1}, \xi\right)\right)\right) \mathrm{d} \xi \\
& +\int_{a_{1}}^{b_{1}}\left(u\left(x, t, \tau, b_{2}\right) F_{2}\left(w\left(\tau, b_{2}\right)\right)-u\left(x, t, \tau, a_{2}\right) F_{2}\left(w\left(\tau, a_{2}\right)\right)\right) \mathrm{d} \tau .
\end{aligned}
$$

We apply this to the Equation (1). For this we write: 


$$
a(\tau, \xi) w_{\tau}(\tau, \xi)+b(\tau, \xi) w_{\xi}(\tau, \xi)=h(\tau, \xi) w(\tau, \xi)+r(\tau, \xi) .
$$

We take as vector field

$$
F^{*}=\left(F_{1}(w), F_{2}(w)\right)=(a(\tau, \xi) w(\tau, \xi), b(\tau, \xi) w(\tau, \xi))
$$

and

$$
u(x, t, \tau, \xi)=\mathrm{e}^{-m_{1}(x+1)(\tau+1)} \mathrm{e}^{-m_{2}(t+1)(\xi+1)}
$$

where $\begin{array}{lllll}m_{1} & \text { y } & m_{2} & \text { are arbitrary constants. Then }\end{array}$

$$
\begin{aligned}
\operatorname{div}\left(F^{*}\right) & =(a(\tau, \xi) w(\tau, \xi))_{\tau}+(b(\tau, \xi) w(\tau, \xi))_{\xi} \\
& =a w_{\tau}+b w_{\xi}+a_{\tau} w+b_{\xi} w=h w+r+a_{\tau} w+b_{\xi} w=h^{*}(w) .
\end{aligned}
$$

Therefore, Equation (8) yields

$$
\int_{a_{1}}^{b_{1}} \int_{a_{2}}^{b_{2}} u w\left(h+a_{\tau}+b_{\xi}-m_{1}(x+1) a-m_{2}(t+1) b\right) \mathrm{d} \xi \mathrm{d} \tau=G(x, t)-\int_{a_{1}}^{b_{1}} \int_{a_{2}}^{b_{2}} u r \mathrm{~d} \xi \mathrm{d} \tau .
$$

\section{Solution of Generalized Moment Problems}

If (9) can be written in the form:

$$
\int_{a_{1}}^{b_{1}} \int_{a_{2}}^{b_{2}} F(w(\tau, \xi)) K(x, t, \tau, \xi) \mathrm{d} \tau \mathrm{d} \xi=\varphi(x, t)
$$

with $\varphi(x, t) \in L^{2}(E)$, then taking a basis $\left\{\psi_{m}(x, t)\right\}_{m}$ of $L^{2}(E)$ this Fredholm integral equation of first kind can be transformed into a bi-dimensional generalized moment problem

$$
\int_{a_{1}}^{b_{1}} \int_{a_{2}}^{b_{2}} F(w(\tau, \xi)) K_{m}(\tau, \xi) \mathrm{d} \tau \mathrm{d} \xi=\mu_{m} \quad m=0,1,2, \cdots
$$

where

$$
K_{m}(\tau, \xi)=\int_{a_{1}}^{b_{1}} \int_{a_{2}}^{b_{2}} K(x, t, \tau, \xi) \psi_{m}(x, t) \mathrm{d} x \mathrm{~d} t
$$

and the moments $\mu_{m}$ are

$$
\mu_{m}=\int_{a_{1}}^{b_{1}} \int_{a_{2}}^{b_{2}} \varphi(x, t) \psi_{m}(x, t) \mathrm{d} x \mathrm{~d} t .
$$

If the functions $\left\{K_{m}(\tau, \xi)\right\}_{m}$ are linearly independent then the generalized moment problem defined by Equations (10), (11) and (12) can be solved considering the correspondent finite problem

$$
\int_{a_{1}}^{b_{1}} \int_{a_{2}}^{b_{2}} F(w(\tau, \xi)) K_{m}(\tau, \xi) \mathrm{d} \tau \mathrm{d} \xi=\mu_{m} \quad m=0,1,2, \cdots, n \quad n \in N
$$

whose solution we denote $p_{n}(\tau, \xi) \approx \beta(\tau, \xi)=F(w(\tau, \xi))$.

If $F(w)$ has continuous inverse, then $F^{-1}\left(p_{n}(\tau, \xi)\right)=w_{n}(\tau, \xi)$ is an estimation of $w(\tau, \xi)$.

To reach this result let consider the basis $\left\{\phi_{i}(\tau, \xi)\right\}_{i=0}^{\infty}$ obtained from the sequence $\left\{K_{m}(\tau, \xi)\right\}_{m=0}^{n}$ by Gram-Schmidt method and addition of the necessary functions in order to have an orthonormal basis.

We then approximate the solution $\beta(\tau, \xi)=F(w(\tau, \xi))$ de (13) with

$$
p_{n}(\tau, \xi)=\sum_{i=0}^{n} \lambda_{i} \phi_{i}(\tau, \xi)
$$

with

$$
\lambda_{i}=\sum_{j=0}^{i} C_{i j} \mu_{j} \quad i=0,1, \cdots, n
$$

where the coefficients $C_{i j}$ verifies 


$$
\begin{aligned}
C_{i j} & =\left(\sum_{k=j}^{i-1}(-1) \frac{\left\langle K_{i}(\tau, \xi) \mid \phi_{k}(\tau, \xi)\right\rangle}{\left\|\phi_{k}(\tau, \xi)\right\|^{2}} C_{k j}\right) \cdot\left\|\phi_{i}(\tau, \xi)\right\|^{-1} \quad 1<i \leq n ; 1 \leq j<i \\
C_{i i} & =\left\|\phi_{i}(\tau, \xi)\right\|^{-1} \quad i=0,1, \cdots, n .
\end{aligned}
$$

We extend to the bi-dimensional case the arguments of reference [8] [9] and we have the following.

Theorem 1. Let $\left\{\mu_{m}\right\}_{m=0}^{n}$ be a set of real numbers and let $\varepsilon$ and $E$ be two positive numbers such that

$$
\sum_{m=0}^{n}\left|\int_{a_{2}}^{b_{2}} \int_{a_{1}}^{b_{1}} K_{m}(\tau, \xi) \beta(\tau, \xi) \mathrm{d} \tau \mathrm{d} \xi-\mu_{m}\right|^{2} \leq \varepsilon^{2}
$$

$y$

then

$$
\int_{a_{2}}^{b_{2}} \int_{a_{1}}^{b_{1}}\left[\left(b_{1}-a_{1}\right)^{2} \beta_{\tau}^{2}+\left(b_{2}-a_{2}\right)^{2} \beta_{\xi}^{2}\right] \mathrm{d} \tau \mathrm{d} \xi \leq E^{2}
$$

$$
\int_{a_{2}}^{b_{2}} \int_{a_{1}}^{b_{1}}|\beta(\tau, \xi)|^{2} \mathrm{~d} \tau \mathrm{d} \xi \leq \min _{n}\left\{\left\|C C^{\mathrm{T}}\right\| \varepsilon^{2}+\frac{E^{2}}{8(n+1)^{2}} ; n=0,1, \cdots, N\right\}
$$

where $C$ is the triangular matriz with elements $C_{i j} \quad(1<i \leq n ; 1 \leq j<i)$.

And

$$
\int_{a_{2}}^{b_{2}} \int_{a_{1}}^{b_{1}}\left|p_{n}(\tau, \xi)-\beta(\tau, \xi)\right|^{2} \mathrm{~d} \tau \mathrm{d} \xi \leq\left\|C C^{\mathrm{T}}\right\| \varepsilon^{2}+\frac{E^{2}}{8(n+1)^{2}} .
$$

Si $F^{-1}(x)$ is Lipschitz in $R^{2}$, ie if $\left\|F^{-1}(x)-F^{-1}(y)\right\| \leq \lambda\|x-y\|$ for some $\lambda$ and $\forall(x, y) \in R^{2}$ then

$$
\int_{a_{2}}^{b_{2}} \int_{a_{1}}^{b_{1}}\left|w_{n}(\tau, \xi)-w(\tau, \xi)\right|^{2} \mathrm{~d} \tau \mathrm{d} \xi \leq \lambda\left(\left\|C C^{\mathrm{T}}\right\| \varepsilon^{2}+\frac{E^{2}}{8(n+1)^{2}}\right) .
$$

Proof. The demonstration is similar to that we have done for the unidimensional generalized moment problem [8], which is based in results of Talenti [10] for the Hausdorff moment problem. Here we simply introduce the necessary modification for the bi-dimensional case.

Without loss of generality we take $\left\{\mu_{m}=0\right\}_{m=0}^{n}$ in (16).

We write

$$
\beta(\tau, \xi)=h_{n}(\tau, \xi)+t_{n}(\tau, \xi)
$$

where $h_{n}(\tau, \xi)$ is the orthogonal projection of $\beta(\tau, \xi)$ on the linear space that the set $\left\{K_{m}(\tau, \xi)\right\}_{m=0}^{n}$ generates and $t_{n}(\tau, \xi)=\beta(\tau, \xi)-h_{n}(\tau, \xi)$ is the orthogonal projection of $\beta(\tau, \xi)$ on the orthogonal complement. In terms of the basis $\left\{\phi_{i}(\tau, \xi)\right\}_{i=0}^{\infty}$ the functions $h_{n}(\tau, \xi)$ and $t_{n}(\tau, \xi)$ reads

with

$$
h_{n}(\tau, \xi)=\sum_{i=0}^{n} \lambda_{i} \phi_{i}(\tau, \xi) ; \quad t_{n}(\tau, \xi)=\sum_{i=n+1}^{\infty} \lambda_{i} \phi_{i}(\tau, \xi)
$$

$$
\lambda_{i}=\sum_{j=0}^{i} C_{i j} \mu_{j} \quad i=0,1, \cdots
$$

and the matrix elements $C_{i j}$ given by (14) and (15).

In matricial notation:

$$
\lambda=\left(\begin{array}{c}
\lambda_{1} \\
\lambda_{2} \\
\vdots \\
\lambda_{n}
\end{array}\right) \quad \mu=\left(\begin{array}{c}
\mu_{1} \\
\mu_{2} \\
\vdots \\
\mu_{n}
\end{array}\right) \quad \Rightarrow \lambda=C \mu
$$


Besides

$$
\lambda_{i}=\int_{a_{2}}^{b_{2}} \int_{a_{1}}^{b_{1}} \beta(\tau, \xi) \phi_{i}(\tau, \xi) \mathrm{d} \tau \mathrm{d} \xi \quad \text { y } \quad \mu_{i}=\int_{a_{2}}^{b_{2}} \int_{a_{1}}^{b_{1}} \beta(\tau, \xi) K_{i}(\tau, \xi) \mathrm{d} \tau \mathrm{d} \xi
$$

Therefore

$$
\int_{a_{2}}^{b_{2}} \int_{a_{1}}^{b_{1}}\left|h_{n}(\tau, \xi)\right|^{2} \mathrm{~d} \tau \mathrm{d} \xi=\langle\lambda, \lambda\rangle=\left\langle C^{\mathrm{T}} C \mu, \mu\right\rangle \leq\left\|C^{\mathrm{T}} C\right\|\|\mu\|^{2} \leq\left\|C^{\mathrm{T}} C\right\| \varepsilon^{2} .
$$

To estimate the norm of $t_{n}(\tau, \xi)$ we observe that each element of the orthonormal basis $\left\{\phi_{i}(\tau, \xi)\right\}_{i=0}^{\infty}$ can be written as a function of the elements of another orthonormal basis, in particular the set $\left\{P_{k l}(\tau, \xi)\right\}_{k, l=0}^{\infty}$ con $P_{k l}(\tau, \xi)=L_{1 k}(\tau) L_{2 l}(\xi)$ with $L_{1 k}(\tau)$ Legendre polynomial in $\left(a_{1}, b_{1}\right), L_{2 l}(\xi)$ Legendre polynomial in $\left(a_{2}, b_{2}\right)$

$$
\phi_{i}(\tau, \xi)=\sum_{k=0}^{\infty} \sum_{l=0}^{\infty} \gamma_{k l, i} P_{k l}(\tau, \xi)
$$

The Legendre polynomials $L_{1 k}(\tau)$ verify

$$
\frac{\mathrm{d}}{\mathrm{d} \tau}\left[\left(a_{1}-\tau\right)\left(b_{1}-\tau\right) L_{1 k}(\tau)\right]=k(k+1) L_{1 k}(\tau) \quad k=0,1,2, \cdots
$$

and analogous property for the polynomials $L_{2 l}(\xi)$.

Defining $\lambda_{k l}^{*}=\sum_{i=n+1}^{\infty} \lambda_{i} \gamma_{k l, i}$ we can demonstrate that

$$
\int_{a_{2}}^{b_{2}} \int_{a_{1}}^{b_{1}}\left|t_{n}(\tau, \xi)\right|^{2} \mathrm{~d} \tau \mathrm{d} \xi \leq \sum_{k=0}^{\infty} \sum_{l=0}^{\infty} k(k+1) \lambda_{k l}^{* 2} \leq \frac{1}{4(n+1)^{2}} \int_{a_{2}}^{b_{2}} \int_{a_{1}}^{b_{1}}\left(b_{1}-a_{1}\right)^{2} \beta_{\tau}^{2}(\tau, \xi) \mathrm{d} \tau \mathrm{d} \xi
$$

and

$$
\int_{a_{2}}^{b_{2}} \int_{a_{1}}^{b_{1}}\left|t_{n}(\tau, \xi)\right|^{2} \mathrm{~d} \tau \mathrm{d} \xi \leq \sum_{k=0 l=0}^{\infty} \sum^{\infty} l(l+1) \lambda_{k l}^{* 2} \leq \frac{1}{4(n+1)^{2}} \int_{a_{2}}^{b_{2}} \int_{a_{1}}^{b_{1}}\left(b_{2}-a_{2}\right)^{2} \beta_{\xi}^{2}(\tau, \xi) \mathrm{d} \tau \mathrm{d} \xi .
$$

From these equations we deduce that

$$
\begin{aligned}
& \int_{a_{2}}^{b_{2}} \int_{a_{1}}^{b_{1}}\left|t_{n}(\tau, \xi)\right|^{2} \mathrm{~d} \tau \mathrm{d} \xi \leq \frac{1}{8(n+1)^{2}} \int_{a_{2}}^{b_{2}} \int_{a_{1}}^{b_{1}}\left[\left(b_{1}-a_{1}\right)^{2} \beta_{\tau}^{2}(\tau, \xi)+\left(b_{2}-a_{2}\right)^{2} \beta_{\xi}^{2}(\tau, \xi)\right] \mathrm{d} \tau \mathrm{d} \xi \\
& \therefore \int_{a_{2}}^{b_{2}} \int_{a_{1}}^{b_{1}}\left|t_{n}(\tau, \xi)\right|^{2} \mathrm{~d} \tau \mathrm{d} \xi \leq \frac{E^{2}}{8(n+1)^{2}} .
\end{aligned}
$$

Adding the expressions for the two standards $\left\|h_{n}(\tau, \xi)\right\|$ y $\left\|t_{n}(\tau, \xi)\right\|^{2}$ result (17) is reached. An analogous demonstration proves inequality (18).

\section{Numerical Examples}

Let consider the equation

$$
x t w_{x}+w_{t}=0
$$

in the domain $E=(0,2) \times(0,2)$ and boundary condition on $\partial E$ given by

$$
\begin{array}{cc}
w(0, t)=\mathrm{e}^{-t^{2} / 2} & w(2, t)=3 \mathrm{e}^{-t^{2} / 2} \\
w(x, 0)=(1+x) & w(x, 2)=(1+x) \mathrm{e}^{-2}
\end{array}
$$

The exact solution is $w(x, t)=(x+1) \mathrm{e}^{-t^{2} / 2}$.

In Figure 1(a) the approximate numerical solution (dark gray) and the exact one (light gray) are compared. Was taken $\psi_{i j}(x, t)=x^{i} t^{j}$ with $i=0,1,2$ and $j=0,1,2$.

And $m_{1}=m_{2}=1$ in $u$. 


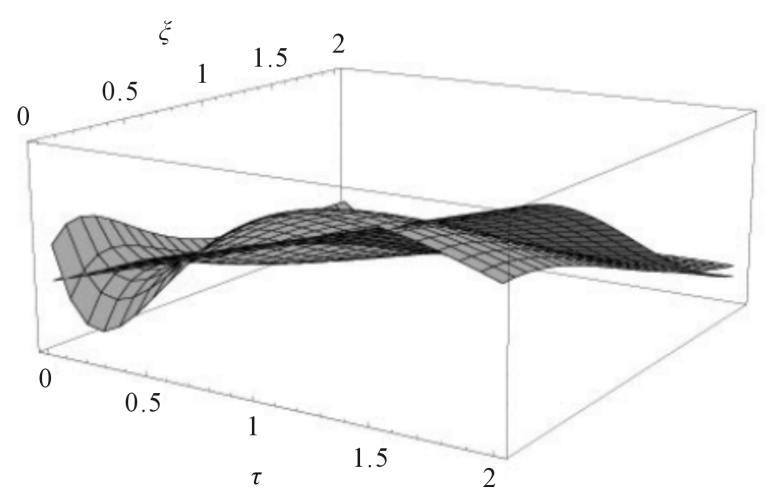

(a)

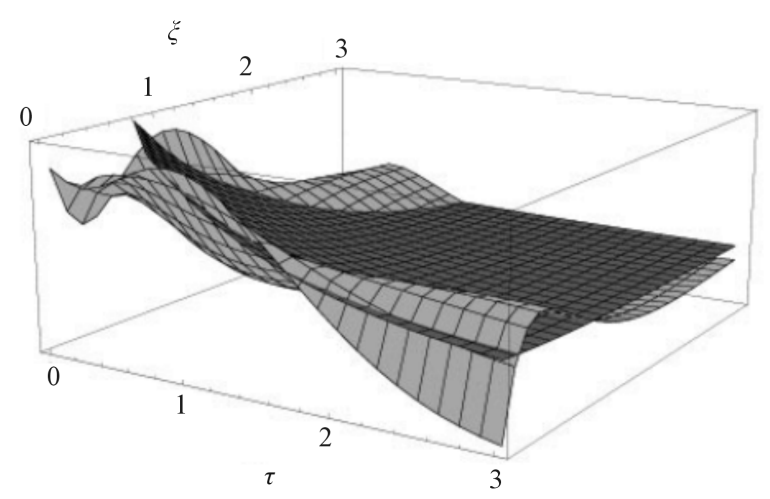

(b)

Figure 1. (a) $w(x, t)=(x+1) \mathrm{e}^{-t^{2} / 2}$; (b) $w(x, t)=\mathrm{e}^{-x-t}$.

Thus were taken $n=9$ moments.

The accuracy is, in this case $\int_{0}^{2} \int_{0}^{2}\left|p_{9}(x, t)-w(x, t)\right|^{2} \mathrm{~d} x \mathrm{~d} t=0.437848$.

Let consider the equation

$$
\left(x t+t^{2}\right) w_{x}+\left(x^{2}+t\right) w_{t}=-(t(1+t)+x(t+x)) w
$$

in the domain $E=(0,3) \times(0,3)$ and boundary condition on $\partial E$ given by

$$
\begin{array}{cc}
w(0, t)=\mathrm{e}^{-t} & w(3, t)=\mathrm{e}^{-3-t} \\
w(x, 0)=\mathrm{e}^{-x} & w(x, 3)=\mathrm{e}^{-3-x}
\end{array}
$$

The exact solution is $w(x, t)=\mathrm{e}^{-x-t}$.

In Figure 1(b) the approximate numerical solution (dark gray) and the exact one (light gray) are compared.

Was taken $\psi_{i j}(x, t)=x^{i} t^{j}$ with $i=0,1,2$ and $j=0,1,2$.

And $m_{1}=m_{2}=1$ in $u$.

Thus were taken $n=9$ moments.

The accuracy is, in this case $\int_{0}^{3} \int_{0}^{3}\left|p_{9}(x, t)-w(x, t)\right|^{2} \mathrm{~d} x \mathrm{~d} t=0.333021$.

\section{Application}

We want to find $w(x, t) \in L^{2}(E)$ with $E=\left(a_{1}, b_{1}\right) \times\left(a_{2}, b_{2}\right)$ such that satisfies the Klein-Gordon equation

$$
w_{x x}(x, t)-w_{t t}(x, t)=h(w(x, t))+r(x, t)
$$

where $h$ y $r$ are known functions.

And boundary conditions

we write

$$
\begin{aligned}
w\left(a_{1}, t\right)=g_{1}(t) & w\left(b_{1}, t\right)=g_{2}(t) \\
w\left(x, a_{2}\right)=g_{3}(x) & w\left(x, b_{2}\right)=g_{4}(x)
\end{aligned}
$$

$$
w_{\tau \tau}(\tau, \xi)-w_{\xi \xi}(\tau, \xi)=h(w(\tau, \xi))+r(\tau, \xi)
$$

We take as vector field

$$
F^{*}=\left(F_{1}(w), F_{2}(w)\right)=\left(w_{\tau}(\tau, \xi),-w_{\xi}(\tau, \xi)\right)
$$

and

$$
u(x, t, \tau, \xi)=\mathrm{e}^{-(x+1)(\tau+1)} \mathrm{e}^{-(t+1)(\xi+1)} .
$$


Then

$$
\operatorname{div}\left(F^{*}\right)=w_{\tau \tau}-w_{\xi \xi}=h^{*}(\tau, \xi)
$$

where

$$
h^{*}(\tau, \xi)=h(w(\tau, \xi))+r(\tau, \xi)
$$

Since

we have

$$
\operatorname{udiv}\left(F^{*}\right)=u h^{*}(w)
$$

$$
\iint_{E} u \operatorname{div}\left(F^{*}\right) \mathrm{d} A=\iint_{E} u h^{*}(w) \mathrm{d} A
$$

Moreover, as

$$
u \operatorname{div}\left(F^{*}\right)=\operatorname{div}\left(u F^{*}\right)-F^{*} \cdot \nabla u
$$

Therefore

$$
\iint_{E} u \operatorname{div}\left(F^{*}\right) \mathrm{d} A=\iint_{E} \operatorname{div}\left(u F^{*}\right) \mathrm{d} A-\iint_{E} F^{*} \cdot \nabla u \mathrm{~d} A
$$

in addition

$$
\iint_{E} \operatorname{div}\left(u F^{*}\right) \mathrm{d} A=\iint_{E}\left(\left(u w_{\tau}\right)_{\tau}-\left(u w_{\xi}\right)_{\xi}\right)=\iint_{E} u \operatorname{div}\left(F^{*}\right) \mathrm{d} A+\iint_{E}\left(u_{\tau} w_{\tau}-u_{\xi} w_{\xi}\right) \mathrm{d} A
$$

then (22) and (23) we obtain:

$$
\iint_{E} F^{*} \cdot \nabla u \mathrm{~d} A=\iint_{E}\left(u_{\tau} w_{\tau}-u_{\xi} w_{\xi}\right) \mathrm{d} A .
$$

Also doing integration by parts is reached:

$$
\iint_{E} F^{*} \cdot \nabla u d A=A(x, t)+B(x, t)-\iint_{E} u w\left((x+1)^{2}-(t+1)^{2}\right) \mathrm{d} A
$$

with

$$
\begin{aligned}
& A(x, t)=\int_{a_{2}}^{b_{2}}\left(w\left(b_{1}, \xi\right) u_{\tau}\left(x, t, b_{1}, \xi\right)-w\left(a_{1}, \xi\right) u_{\tau}\left(x, t, a_{1}, \xi\right)\right) \mathrm{d} \xi \\
& B(x, t)=\int_{a_{1}}^{b_{1}}\left(w\left(\tau, b_{2}\right) u_{\xi}\left(x, t, \tau, b_{2}\right)-w\left(\tau, a_{2}\right) u_{\xi}\left(x, t, \tau, a_{2}\right)\right) \mathrm{d} \tau .
\end{aligned}
$$

From (23), (24) and (25) and after several calculations:

$$
A(x, t)+B(x, t)=\int_{a_{2}}^{b_{2}} \int_{a_{1}}^{b_{1}} u\left[w\left[(x+1)^{2}-(t+1)^{2}\right]+w_{\tau}(-1-x)+w_{\xi}(1+t)\right] \mathrm{d} x \mathrm{~d} t .
$$

If $t=x$ then

$$
\varphi(t)=A(t, t)+B(t, t)=\int_{a_{2}}^{b_{2}} \int_{a_{1}}^{b_{1}} u(1+t)\left[-w_{\tau}+w_{\xi}\right] \mathrm{d} x \mathrm{~d} t .
$$

We write (26) as:

$$
\frac{\varphi(t)}{(1+t)}=\int_{a_{2}}^{b_{2}} \int_{a_{1}}^{b_{1}} \mathrm{e}^{-(t+1)(\tau+\xi+2)}\left[-w_{\tau}+w_{\xi}\right] \mathrm{d} x \mathrm{~d} t .
$$

We can see that (27) is an integral equation of the form

$$
\int_{a_{1}}^{b_{1}} \int_{a_{2}}^{b_{2}} w^{*}(\tau, \xi) K(t, \tau, \xi) \mathrm{d} \tau \mathrm{d} \xi=\varphi^{*}(t)
$$

where the unknown function is $w^{*}(\tau, \xi)=-w_{\tau}+w_{\xi}$, the kernel is $K(t, \tau, \xi)=\mathrm{e}^{-(t+1)(\tau+\xi+2)}$ and $\varphi^{*}(t)=\frac{\varphi(t)}{(1+t)}$. 
To solve (27) as a problem of two-dimensional moments we apply seen in Section 3 and we obtain an approximation $p_{n}(\tau, \xi)$ to $-w_{\tau}+w_{\xi}$.

Now we solve the partial differential equation of the first order

$$
-w_{\tau}+w_{\xi}=r(\tau, \xi)
$$

where $r(\tau, \xi)=p_{n}(\tau, \xi), a(\tau, \xi)=-1, b(\tau, \xi)=1$ y $h(\tau, \xi)=0$.

To find the solution of the Equation (28) algorithm of Section 3 applies.

\section{Numerical Examples}

We want to find $w(x, t) \in L^{2}(E)$ with $E=(0,2) \times(0, \infty)$ such that satisfies the Klein-Gordon equation

$$
w_{t t}(x, t)-w_{x x}(x, t)=\left(4 t^{2}+8 t+1\right) w(x, t)+2 \mathrm{e}^{-x-(t+1)^{2}}
$$

with boundary conditions

$$
\begin{gathered}
w(0, t)=\mathrm{e}^{-(t+1)^{2}} \quad w(2, t)=3 \mathrm{e}^{-2-(t+1)^{2}} \quad t>0 \\
w(x, 0)=(x+1) \mathrm{e}^{-x}, \quad 0<x<2 .
\end{gathered}
$$

The exact solution is $w(x, t)=(x+1) \mathrm{e}^{-x-(t+1)^{2}}$.

In Figure 2(a) the approximate numerical solution (dark grey) and the exact one (light grey) are compared.

For the first step was taken the base $\psi_{i}(t)=t^{i} \mathrm{e}^{-t}$ with $i=0,1, \cdots, 8$ and as an auxiliary function

$u(x, t, \tau, \xi)=\mathrm{e}^{-x(1+\xi)-t(1+\tau)}$. For the second step was taken the base $\psi_{i j}(x, t)=x^{i} t^{j} \mathrm{e}^{-x-t}$ with $i=0,1,2$ and $j=0,1,2$.

And $u(x, t, \tau, \xi)=\mathrm{e}^{-(x+1)(1+\xi)-0.5(t+1)(1+\tau)}$ in order to avoid discontinuities.

Thus were taken $n=9$ moments.

The accuracy is, in this case $\int_{0}^{\infty} \int_{0}^{2}\left|p_{9}(x, t)-w(x, t)\right|^{2} \mathrm{~d} x \mathrm{~d} t=0.0997437$.

We want to find $w(x, t) \in L^{2}(E)$ with $E=(0,2) \times(0,2)$ such that satisfies the Klein-Gordon equation

$$
w_{t t}(x, t)-w_{x x}(x, t)=-\mathrm{e}^{w}
$$

with boundary conditions

$$
\begin{array}{cc}
w(0, t)=\ln \left(\frac{6}{(t+3)^{2}}\right) & w(2, t)=\ln \left(\frac{6}{(t+7)^{2}}\right) \\
w(x, 0)=\ln \left(\frac{6}{(2(x+1)+1)^{2}}\right), & w(x, 2)=\ln \left(\frac{6}{(2(x+1)+3)^{2}}\right)
\end{array}
$$

The exact solution is $w(x, t)=\ln \left(\frac{6}{(2(x+1)+t+1)^{2}}\right)$.

In Figure 2(b) the approximate numerical solution (dark grey) and the exact one (light grey) are compared.

For the first step was taken the base $\psi_{i}(t)=t^{i} \mathrm{e}^{-t}$ with $i=0,1, \cdots, 8$ and as an auxiliary function

$u(x, t, \tau, \xi)=\mathrm{e}^{-x(1+\xi)-t(1+\tau)}$. For the second step was taken the base $\psi_{i j}(x, t)=x^{i} t^{j} \mathrm{e}^{-x-t}$ with $i=0,1,2$ and $j=0,1,2$.

And $u(x, t, \tau, \xi)=\mathrm{e}^{-(x+1)(1+\xi)-0.5(t+1)(1+\tau)}$ in order to avoid discontinuities.

Thus were taken $n=9$ moments.

The accuracy is, in this case $\int_{0}^{2} \int_{0}^{2}\left|p_{9}(x, t)-w(x, t)\right|^{2} \mathrm{~d} x \mathrm{~d} t=0.615126$.

\section{Conclusions}

The linear partial differential equations of first order 


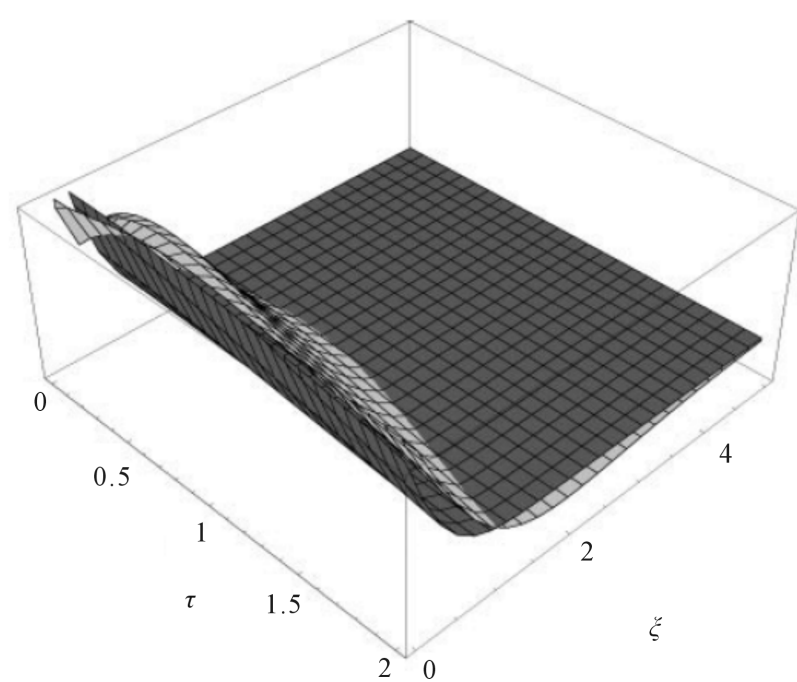

(a)

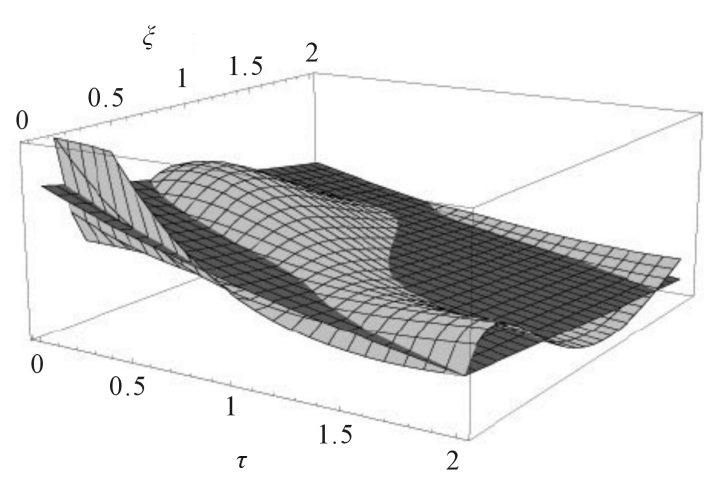

(b)

Figure 2. (a) $w(x, t)=(x+1) \mathrm{e}^{-x-(t+1)^{2}}$, (b) $w(x, t)=\ln \left(\frac{6}{(2(x+1)+t+1)^{2}}\right)$.

$$
a(x, t) w_{x}(x, t)+b(x, t) w_{t}(x, t)=h(x, t) w(x, t)+r(x, t)
$$

on a region $E=\left(a_{1}, b_{1}\right) \times\left(a_{2}, b_{2}\right)$ can be written as an Fredholm integral equation

$$
\int_{a_{1}}^{b_{1}} \int_{a_{2}}^{b_{2}} u w\left(h+a_{\tau}+b_{\xi}-m_{1}(x+1) a-m_{2}(t+1) b\right) \mathrm{d} \xi \mathrm{d} \tau=G(x, t)-\int_{a_{1}}^{b_{1}} \int_{a_{2}}^{b_{2}} u r \mathrm{~d} \xi \mathrm{d} \tau .
$$

If (31) can be written in the form:

$$
\int_{a_{1}}^{b_{1}} \int_{a_{2}}^{b_{2}} F(w(\tau, \xi)) K(x, t, \tau, \xi) \mathrm{d} \tau \mathrm{d} \xi=\varphi(x, t)
$$

with $\varphi(x, t) \in L^{2}(E)$, then taking a basis $\left\{\psi_{m}(x, t)\right\}_{m}$ of $L^{2}(E)$ this Fredholm integral equation of the first kind can be transformed into a bi-dimensional generalized moment problem

$$
\int_{a_{1}}^{b_{1}} \int_{a_{2}}^{b_{2}} F(w(\tau, \xi)) K_{m}(\tau, \xi) \mathrm{d} \tau \mathrm{d} \xi=\mu_{m} \quad m=0,1,2, \cdots
$$

where

$$
K_{m}(\tau, \xi)=\int_{a_{1}}^{b_{1}} \int_{a_{2}}^{b_{2}} K(x, t, \tau, \xi) \psi_{m}(x, t) \mathrm{d} x \mathrm{~d} t
$$

and the moments $\mu_{m}$ are

$$
\mu_{m}=\int_{a_{1}}^{b_{1}} \int_{a_{2}}^{b_{2}} \varphi(x, t) \psi_{m}(x, t) \mathrm{d} x \mathrm{~d} t .
$$

If the functions $\left\{K_{m}(\tau, \xi)\right\}_{m}$ are linearly independent then the generalized moment problem defined by Equations (32), (33) and (34) can be solved considering the correspondent finite problem.

\section{References}

[1] Akheizer, N.I. (1965) The Classical Moment Problem. Olivier and Boyd, Edinburgh.

[2] Akheizer, N.I. and Krein, M.G. (1962) Some Questions in the Theory of Moment. American Mathematical Society. Providence.

[3] Shohat, J.A. and Tamarkin, J.D. (1943) The Problem of Moments. Mathematical Surveys, American Mathematical Society, Providence. http://dx.doi.org/10.1090/surv/001

[4] Ang, D.D., Gorenflo, R., Le, V.K. and Trong, D.D. (2002) Moment Theory and Some Inverse Problems in Potential 
Theory and Heat Conduction. Lectures Notes in Mathematics, Springer-Verlag, Berlin. http://dx.doi.org/10.1007/b84019

[5] Pintarelli, M.B. and Vericat, F. (2012) Klein-Gordon Equation as a Bi-Dimensional Moment Problem. Far East Journal of Mathematical Sciences, 70, 201-225.

[6] Tikhonov, A. and Arsenine, V. (1976) Méthodes de résolution de problèmes mal posés. MIR, Moscow.

[7] Engl, H.W. and Groetsch, C.W. (1987) Inverse and Ill-Posed Problems. Academic Press, Boston.

[8] Pintarelli, M.B. and Vericat, F. (2008) Stability Theorem and Inversion Algorithm for a Generalized Moment Problem. Far East Journal of Mathematical Sciences, 30, 253-274.

[9] Pintarelli, M.B. and Vericat, F. (2011) Bi-Dimensional Inverse Moment Problems. Far East Journal of Mathematical Sciences, 54, 1-23.

[10] Talenti, G. (1987) Recovering a Function from a Finite Number of Moments. Inverse Problems, 3, 501-517. http://dx.doi.org/10.1088/0266-5611/3/3/016

[11] Ames, W.F. (1992) Numerical Methods for Partial Differential Equations. Academic Press, New York.

[12] Lapidus, L. and Pinder, G.F. (1982) Numerical Solution of Partial Differential Equations in Science and Engineering. John Wiley and Sons, New York.

[13] Smith, G.D. (1985) Numerical Solution of Partial Differential Equations: Finite Difference Methods. Oxford University Press, New York.

[14] Thomas, J.W. (1995) Numerical Partial Differential Equations: Finite Difference Methods. Springer-Verlag, New York. http://dx.doi.org/10.1007/978-1-4899-7278-1 